\title{
ADHD: a hidden comorbidity in adult psychiatric patients
}

\author{
István Bitter ${ }^{1}$ • Pavel Mohr ${ }^{2,3} \cdot$ Lívia Balogh $^{1} \cdot$ Klára Látalová $^{4} \cdot$ Brigitta Kakuszi $^{1} \cdot$ Pavla Stopková2,3 $^{2}$ \\ Daniela Zmeškalová-Jelenová ${ }^{4}$. Attila Pulay ${ }^{1} \cdot$ Pál Czobor $^{1}$
}

Received: 26 June 2018 / Accepted: 8 January 2019

(c) The Author(s) 2019

\begin{abstract}
Adult attention-deficit/hyperactivity disorder (aADHD) has recently been better recognized and treated in many European countries. In spite of this development, aADHD still features as a "hidden" comorbidity, often not diagnosed even in patients under psychiatric treatment for other psychiatric disorders. The aim of this study was to establish the prevalence rates of unrecognized aADHD in academic centers providing regular psychiatric services in the Czech Republic and Hungary. In a population of psychiatric in-and outpatients, Adult ADHD Self-Report Scale was administered. All positively and about half of the negatively screened subjects were clinically interviewed and the DSM diagnosis of ADHD was determined based on the symptom list and Conners' Adult ADHD Rating Scale. The estimated point prevalence rate of unrecognized comorbid aADHD among psychiatric in-and out patients was 6.99\% (95\% lower CI: 5.11, 95\% upper CI 8.86) according to the DSMIV-TR criteria and 9.27\% (95\% lower CI: 7.13, 95\% upper CI 11.40) according to the DSM-5 criteria. Current suicide risk was significantly associated with the presence of undiagnosed aADHD; however, life time suicide attempts, depression, dysthymia, alcohol and substance dependence, anxiety and stress related disorders were not. Further educational efforts are needed to improve the recognition and treatment of aADHD in adults
\end{abstract}

Keywords ADHD $\cdot$ Adult ADHD $\cdot$ Comorbidity $\cdot$ Prevalence $\cdot$ Epidemiology

\section{Introduction}

Attention-deficit/hyperactivity disorder (ADHD) is a neurodevelopmental disorder, characterized with persistent and developmentally inappropriate level of inattention and/or hyperactivity and impulsivity, resulting in functional impairment (Buitelaar et al. 2011). Approximately two-thirds of individuals diagnosed with ADHD early in their lives suffer from symptoms in adult age, as well; $15 \%$ of them meet full

István Bitter and Pavel Mohr have contributed equally.

István Bitter

bitter.istvan@med.semmelweis-univ.hu

1 Department of Psychiatry and Psychotherapy, Semmelweis University, Budapest, Hungary

2 National Institute of Mental Health, Klecany, Czech Republic

3 Third Faculty of Medicine, Charles University in Prague, Prague, Czech Republic

4 Department of Psychiatry, University Hospital Olomouc and Faculty of Medicine and Dentistry, Palacky University Olomouc, Olomouc, Czech Republic
DSM-IV-TR (American Psychiatric Association 2000) diagnostic criteria and the rest is regarded as 'ADHD in partial remission' (Ramos-Quiroga et al. 2013). Typically, the most frequent subtype of adult ADHD (aADHD) is predominantly inattentive type, with associated disorganization, emotional dysregulation (Kooij et al. 2010).

A meta-analysis reported a $2.5 \%$ prevalence of aADHD based on pooled data of population-based studies (Simon et al. 2009). Similar number (current DSM-IV-TR aADHD prevalence of $2.8 \%$ ) has been recently reported in a representative WHO World Mental Health Survey across 20 countries (Fayyad et al. 2017).

Adult ADHD is often associated with negative outcome, as evidenced by impaired psychosocial functioning and low quality of life, including poor academic and occupational performance, interpersonal relationships, driving skills, low socioeconomic status, self-esteem, or high criminality rates (Kooij et al. 2010; Buitelaar et al. 2011). The estimated economic impact of aADHD in the US is \$105-194 billion per year, the highest proportion being attributed to productivity and income loss, \$87-137 billion (Doshi et al. 2012). Swedish register and population-based data suggest that any 
drug treatment of aADHD may reduce criminality, serious traffic accidents, and even suicide rates (Lichtenstein et al. 2012; Chang et al. 2014; Chen et al. 2014).

However, the recognition of aADHD remains poor, which is partly explained by the high rates of comorbidity, which may make the diagnosis difficult (Asherson et al. 2007, 2014). In general, psychiatric comorbidity rates in aADHD can be as high as $49 \%$ for any current and $89 \%$ for any past DSM-IV-TR Axis I disorder, and $78.5 \%$ for at least one, and $45 \%$ for at least two DSM-IV-TR Axis II disorders (Jacob et al. 2007; Sobanski et al. 2007; Cumyn et al. 2009). Specifically, compared with non-ADHD subjects, significantly higher rates of comorbid major depression (MD), dysthymia, bipolar disorder (BD), anxiety disorders, substance use disorders (SUD), personality disorders (PD) were consistently reported in adults with ADHD (Jacob et al. 2007; Sobanski et al. 2007; Cumyn et al. 2009; Asherson et al. 2014; Perroud et al. 2014).

In a complementary manner, some authors suggested that undiagnosed aADHD could be a frequent comorbidity in patients already in treatment for at least one psychiatric disorder other than ADHD (Rao and Place 2011; Ginsberg et al. 2014). In a recent cross-sectional study conducted in psychiatric outpatient health care facilities in eight European countries, the prevalence of aADHD was estimated between 14.2 and $19.0 \%$ (min and max values of $95 \%$ CIs of the prevalence values based on different definitions) (Deberdt et al. 2015).

The primary objective of our study was to estimate the prevalence of undiagnosed aADHD in both inpatient and outpatients of university psychiatric clinics providing "regular" care. The secondary objectives were: (1) to investigate the effect of different diagnostic criteria on prevalence estimates of aADHD assessed in Czech and Hungarian psychiatric inpatient and outpatient samples, and (2) to estimate the rate of comorbidity with aADHD in patients already diagnosed with another psychiatric disorder.

\section{Methods}

The study protocol was approved by the ethics committees for the Department of Psychiatry and Psychotherapy, Semmelweis University in Budapest, Hungary and for the Prague Psychiatric Center/National Institute of Mental Health, Czech Republic. During the course of the study, a third study site (second Czech site) was added, Psychiatric Clinic University Hospital in Olomouc, in order to increase subjects' enrollment. The change was approved by the Ethics Committee.

Eligible study subjects were males and females, age of 18-60 years, currently undergoing inpatient or outpatient psychiatric treatment for a DSM-IV-TR Axis I diagnosis other than ADHD, with capacity to sign informed consent form in their native language. Exclusion criteria were history of major neurological disorders and diagnosis of the following psychiatric disorders: schizophrenia, cognitive disorders (e.g., dementia).

The study consisted of two phases: Phase I (Screening Phase), and Phase II (Interview Phase), described below in detail.

\section{Phase I}

During the Screening Phase, consecutively arriving and consenting patients entered the study on randomly selected days. The number of participants screened during a day varied according to patients consent and availability. Approximately half of the negatively screened participants were approached for to participation in the Interview Phase of the study. All study subjects signed informed consent form prior to administration of any study procedure. A trained assistant distributed to the subjects the ADHD screener, the Adult ADHD Self-Report Scale (ASRS). ASRS is an 18-item self-report scale, based on the symptom list of DSM-IV-TR, developed by the Workgroup on Adult ADHD in conjunction with the World Health Organization (WHO) (Kessler et al. 2007). Symptom frequency was measured on a 5-point Likert scale. In the screening phase of the study, the 6-question, screener version of ASRS was applied. This short version of ASRS was reported to have good sensitivity and specificity as well as predictive value for the diagnosis of aADHD (Kessler et al. 2005). All positively screened and about half of the negatively screened subjects were invited to further participate in the study.

\section{Phase II}

Consenting patients entered Phase II, the Interview Phase. During the clinical interview, in addition to demographic data and diagnosis of aADHD, other potential comorbid psychiatric disorders were assessed.

\section{DSM-IV-TR symptom list}

A structured clinical interview has been earlier developed by the authors, using the symptom list of ADHD in DSMIV-TR, including functional impairment and onset criteria (i.e., whether some of the symptoms had caused problems before the age of seven) as well (Bitter et al. 2010). The interview is comprised of two sections. The first section assessed the presence of ADHD in childhood and included 20 items: 18 symptoms, as well as functional impairment and age of onset. The second section assessed the presence of aADHD based on the same items as in the first section (with the exclusion of age of onset, which has already been 
Table 1 Estimated prevalence rates adjusted for the sensitivity and specificity of the screener and for sex ratio (1:1) for the four diagnostic groups defined in our study

\begin{tabular}{lllcc}
\hline & $\begin{array}{l}\text { ADHD DSM- } \\
\text { IV (full criteria) }\end{array}$ & $\begin{array}{l}\text { ADHD } \\
\text { DSM-IV } \\
\text { Sx }\end{array}$ & $\begin{array}{l}\text { ADHD } \\
\text { DSM-IV no } \\
\text { onset }\end{array}$ & $\begin{array}{l}\text { ADHD DSM-5 } \\
\text { (full criteria) }\end{array}$ \\
\hline Estimated prevalence \% & 6.99 & 13.41 & 9.18 & 9.27 \\
SE for the estimated prevalence & 0.96 & 0.13 & 0.11 & 0.11 \\
$95 \%$ lower CI for the estimated prevalence & 5.11 & 10.90 & 7.06 & 7.13 \\
$95 \%$ upper CI for the estimated prevalence & 8.86 & 15.92 & 11.31 & 11.40 \\
\hline
\end{tabular}

Total number of patients is based on the group of positively screened subjects. Differences in total number of patients is explained by missing data

Table 2 Proportion of DSM-IV ADHD "yes" and "no" diagnoses associated with the most frequent current "main" (comorbid) psychiatric disorders and current suicide risk

\begin{tabular}{|c|c|c|c|c|c|}
\hline \multirow{2}{*}{$\begin{array}{l}\text { Main comorbid psychiatric disorder (MCPD) (MCPD/total number of } \\
\text { available patients) }\end{array}$} & \multirow{2}{*}{$\begin{array}{l}\text { In DSM-IV ADHD } \\
\text { NO }(n) \%\end{array}$} & \multirow{2}{*}{$\begin{array}{l}\text { In DSM-IV ADHD } \\
\text { YES }(n) \%\end{array}$} & \multicolumn{3}{|c|}{ Chi-square } \\
\hline & & & $\mathrm{DF}$ & Value & $P$ \\
\hline Depression $(188 / 438)$ & (171) 42.86 & (17) 43.59 & 1 & 0.01 & 0.93 \\
\hline Suicide risk current $(38 / 433)$ & (10) 54.43 & (28) 73.68 & 1 & 5.21 & 0.02 \\
\hline Alcohol dependence $(65 / 433)$ & (59) 14.97 & (6) 15.38 & 1 & 0.00 & 0.94 \\
\hline Psychoactive substance dependence (other than alcohol) (51/393) & (47) 13.06 & (4) 12.12 & 1 & 0.02 & 0.88 \\
\hline Anorexia (13/432) & (13) 3.31 & (0) 0 & 1 & 1.33 & 0.25 \\
\hline Anxiety and stress related disorders* $(265 / 438)$ & (239) 59.90 & (26) 66.67 & 1 & 0.68 & 0.41 \\
\hline
\end{tabular}

*Generalized anxiety disorder, panic disorder, agoraphobia, social phobia, obsessive-compulsive disorder, posttraumatic stress disorder; none of the listed disorders yielded significant Chi-square results separately

collected in the first section); functional impairment (yes/ no) was established based on whether the symptoms were present and caused problems during the past half a year.

\section{Clinical interview}

During the clinical interview, the interviewer had 20-30 min to collect relevant information about the presence aADHD. The type of information needed to be gathered was the same for all the participants, but the interview was conducted in an open-ended fashion, while the interviewers were making detailed notes. The following issues had to be addressed: complications during pregnancy and delivery; developmental deviations; family background (relationship with parents, siblings, brief family history); preschool nursing (problems with the other children, problems with the preschool teachers, adjustment problems); school years (studies, behavior, relationship with students/teachers); jobs (conflicts with colleagues/supervisors, frequent job and/or workplace changes); relationships.

\section{Demographic and clinical characteristics}

The following items were recorded and included in the assessment of demographic and clinical characteristics: age, gender, years of education, type of education, marital status, current DSM-IV-TR Axis I diagnosis, psychiatric and medical history, family history, medications taken on a regular basis, smoking status, handedness.

\section{Conners' adult ADHD rating scale (CAARS)}

The CAARS 66 item self-report version (CAARS-S:L) developed by Conners et al. (1999) was used. CAARS includes four factor-derived subscales (inattention/memory problems, hyperactivity/restlessness, impulsivity/ emotional lability, problems with self-concept) and three DSM-IV-TR ADHD Subscales: DSM-IV-TR Inattentive Symptoms, DSM-IV-TR Hyperactive-Impulsive Symptoms, DSM-IV-TR Total ADHD Symptoms), the ADHD Index and the Inconsistency Index. Items are rated on a 4 point scale (0-3).

\section{MINI-PLUS 5.0 structured interview}

The validated Czech and Hungarian versions of MINIPLUS structured interview (Balázs et al. 1998; Sheehan et al. 1998) were applied for the assessment of lifetime and current DSM-IV-TR Axis I psychiatric disorders. 


\section{Diagnosis of aADHD}

Based on the documentation of the clinical interview, the study team decided whether the participant meets the criteria for the clinical diagnosis of adult ADHD. Based on previous aADHD prevalence studies (Bitter et al. 2010), modified diagnostic criteria were also applied to investigate the effect of diagnostic criteria on the prevalence estimates. Below, we present three modified criteria based on their relation to the DSM-IV-TR criteria and a fourth criterion based on DSM-5 criteria (American Psychiatric Association 2013).

1. 'ADHD_DSM-IV-TR' diagnostic group: based on the full set of DSM-IV-TR criteria for both childhood and aADHD (combined, or inattentive, or hyperactive/ impulsive type) with supporting information based on the clinical interview.

2. 'ADHD_No-onset' group: based on DSM-IV-TR criteria for both childhood and aADHD (combined or inattentive or hyperactive/impulsive type), excluding onset criterion.

3. 'ADHD_Symptoms-only' group: based on DSM-IV-TR symptom criterion only (6 symptoms had to be present out of the 9 symptoms of either inattention or hyperactivity/impulsivity, or both) for both childhood and aADHD.

4. 'ADHD_DSM-5' diagnostic group: based on the full set of DSM-5 criteria for both childhood and aADHD with supporting information based on the clinical interview. The study protocol was written prior to the publication of DSM-5. DSM-5 criteria were added to the protocol after its publication.

\section{Statistical analysis}

The Statistical Analysis System for Windows (version 9.1; SAS Institute, Cary, NC) was used for statistical analyses. All statistical analyses used the alpha error level of 0.05 (two-sided) and 95\% confidence intervals. In order to determine the true population prevalence, the specificity and sensitivity of the screening test was taken into consideration.

\section{Results}

Seven hundred and sixteen patients were included in the screening phase (Budapest: 580, Prague: 136) between 7th July, 2012 and 26th June, 2014. Eight patients fulfilled one of the exclusion criteria; thus, we report data on 708 patients (Tables 1, 2). Patients disposition including basic demographic data and the ASRS mean scores of the study sample, as well as the 'positively screened', 'interviewed' and 'notinterviewed' and the 'negatively screened' 'interviewed' and 'non-interviewed' subsamples are presented in Fig. 1. Figure 1 also describes the row prevalence rates of the four predefined groups as listed above:

1. 'ADHD_DSM-IV-TR' diagnostic group: based on the full set of DSM-IV-TR criteria for both childhood and aADHD.

2. 'ADHD_No-onset' group: based on DSM-IV-TR criteria for both childhood and AADHD without the criterion of onset.

3. 'ADHD_Symptoms-only' group: based on DSM-IV-TR symptom criterion only (6 symptoms had to be present out of the 9 symptoms of either inattention or hyperactivity/impulsivity, or both) for both childhood and aADHD.

4. 'ADHD_DSM-5' diagnostic group: based on the full set of DSM-5 criteria for both childhood and aADHD.

\section{Discussion}

Our data support earlier findings that aADHD often remains an undiagnosed and consequently untreated disorder even in patients under treatment for another psychiatric disorder (Deberdt et al. 2015). While the estimated point prevalence rates (adjusted for age and sex and for the sensitivity and specificity of the screener) of undiagnosed aADHD in the two Czech and the one Hungarian psychiatric centers were found to be lower than in the cited European study (Deberdt et al. 2015), they are still high. The European study reported row rates "applying DSM-IV-TR-TR or DSM-5 criteria, $15.8 \%$ (95\% confidence interval [CI] $14.2-17.4 \%$ ) or $17.4 \%$ (95\% CI 15.7-19.0\%) of patients were diagnosed with ADHD, respectively", while we report adjusted prevalence estimations for the DSM-IV-TR group 6.99\% (95\% confidence interval [CI] $5.11 \%-8.86 \%$ ) and for the DSM-5 group 9.27\% (95\% confidence interval [CI] 7.13\%-11.40\%). The strength of this study is that in order to improve case identification in addition to ASRS further methods were used, including a semistructured clinical interview, the Conners' Adult ADHD Rating Scale (CAARS) and the MINI-PLUS 5.0 Structured interview. The European study found 10\% higher rates using DSM-5 criteria as compared to DSM-IVTR criteria, while our study shows a $32.6 \%$ higher rate when using DSM-5 criteria as compared to DSM-IV-TR criteria. These results are similar to those of Vitola et al. (2017), who found that "The use of the DSM-5 criteria results in a higher prevalence of ADHD when compared to those obtained by DSM-IV."

We found no significant differences between the sizes of the subgroups with or without DSM-IV-TR aADHD in the investigated diagnostic groups; however, there are statistically significant differences regarding current suicide risk. 


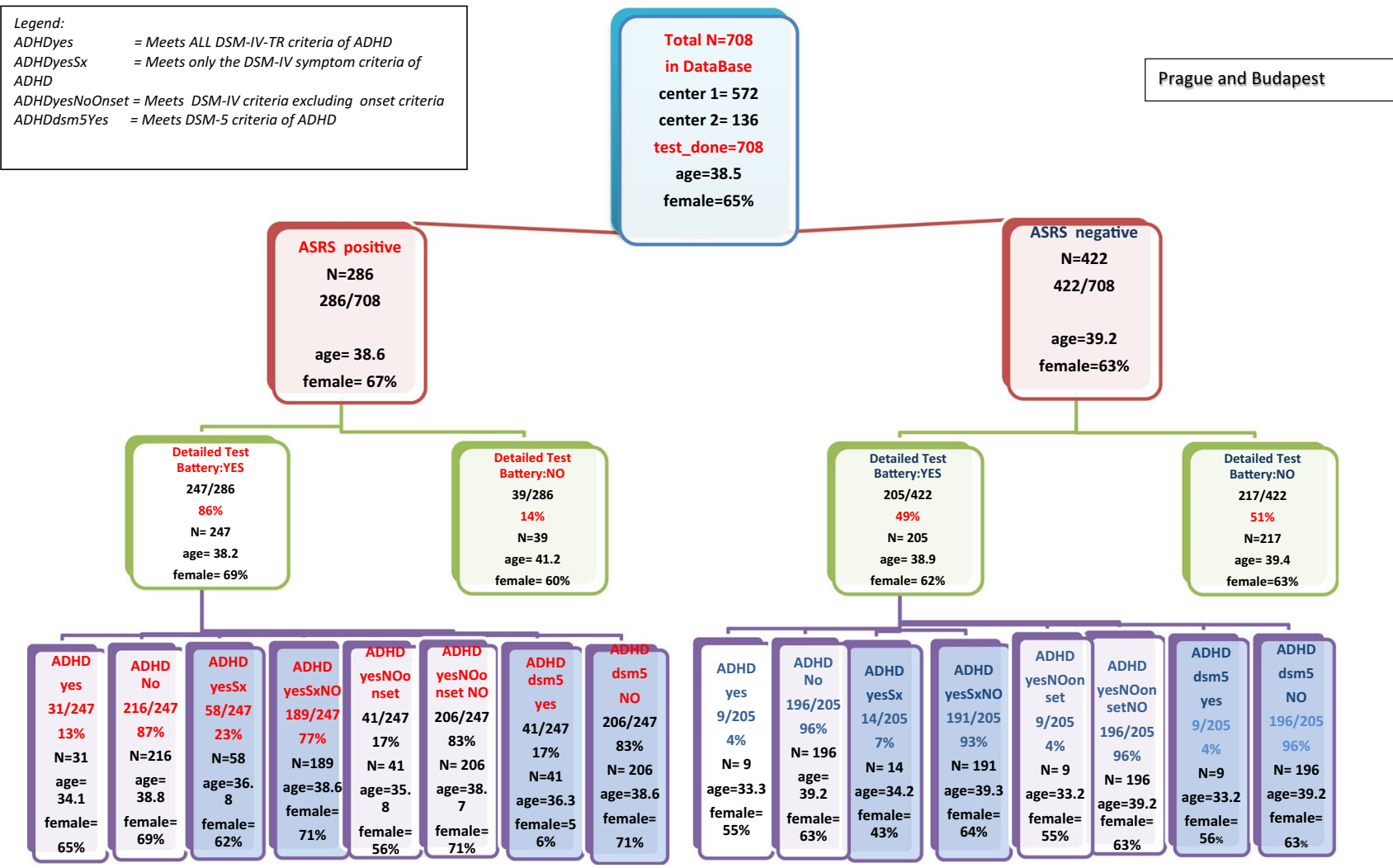

Fig. 1 Study flowchart, basic demographics and raw prevalence rates by patient disposition

This finding is in line with previous studies highlighting the positive association between ADHD and suicidality (Balazs and Kereszteny 2017). The presence of aADHD may be associated with more severe symptoms of the comorbid psychiatric disorders; however, no data were collected in this study to characterize the severity of comorbid disorders.

In case no childhood onset of ADHD can be identified, clinicians should carefully assess impairment, psychiatric history, and substance use before treating potential late-onset cases. False positive late-onset ADHD cases are common without careful assessment (Sibley et al. 2018). However, as Matte et al. (2015) pointed out about the DSM-5 adult ADHD criteria, "The proposed new impulsivity symptoms for adults do not improve ADHD diagnosis enough to overcome potential negative effects of changing the criteria. However, fewer symptoms than the six-of-nine threshold required by DSM-IV provided the best cutoff point for identifying adults who are impaired."

The proper diagnosis and treatment of comorbid ADHD and other psychiatric disorders is important, since they may lead to higher rates of unfavorable outcomes. Available evidence suggests that ADHD is associated with earlier onset of comorbid disorders, such as alcoholism and "ADHD in combination with a comorbid disorder (depression, anxiety disorder, etc.) additionally increases the risk of developing an addiction" (Ohlmeier et al. 2008). Although the presence of comorbid psychiatric conditions with ADHD complicates the treatment and results in more severe dysfunction and impairment (Biederman 2004), the background of the observed high rate of psychiatric comorbidity in ADHD is still an open question. (Sonuga-Barke 2005; Spencer et al. 2007; Biederman et al. 2008; Halmoy et al. 2010).

Developmental disturbances (Sonuga-Barke 2005) as well as the role of overlapping symptoms and possible case over-identification (Halmoy et al. 2010) have already been suggested in the literature. Gender differences were consistently reported in the prevalence of comorbid conditions in adults with ADHD. Specifically, SUD (alcohol use), conduct disorder (CD), antisocial PD were more prevalent among males, while MD, dysthymia, and anxiety disorders were more prevalent among females with ADHD in the majority of the studies (Biederman et al. 1993; Sobanski et al. 2007; Sprafkin et al. 2007; Wilens et al. 2009). Nonetheless, Biederman (2004) reported on no moderating effect of gender on psychiatric comorbidity with ADHD in adults.

Other possible moderating factors for the rate of comorbidity in/with ADHD could be the severity of ADHD and the diagnostic criteria used for ADHD (Bitter et al. 2010). This has been supported by the findings indicating that ADHD symptom severity is significantly correlated with the 
occurrence of lifetime depressive episodes in both males and females (Simon et al. 2013).

Our data underline the need for better training for the recognition of adult ADHD comorbid with substance use and other mental disorders.

\section{Limitations}

The major limitation of this study is its cross-sectional design, which does not permit to establish causal relationships.

Acknowledgements Open access funding provided by Semmelweis University (SE). The authors would like to thank Drs. Anna Angyalosi and Zuzana Kratochvílová, Ágnes Bán Gérecz, Berta Hamza Erdélyi, Enikő Németh and Dóra Uram for their contribution to the data collection and Ms. Márta Kovács for the logistical support.

Funding E. Lilly supported part of this study providing an Investigators Initiated Grant to Drs. Bitter and Mohr. The Sponsor reviewed the proposal and received a copy of the submitted manuscript; however, they had no role in the planning and implementation of the study, drafting and submission of the manuscript or in any other parts of this study.

\section{Compliance with ethical standards}

Conflict of interest The authors declare that beyond the above disclosed funding have no conflict of interest.

Statement on human rights All procedures performed in studies involving human participants were in accordance with the ethical standards of the institutional and/or national research committee and with the 1964 Helsinki declaration and its later amendments or comparable ethical standards. The protocol and the patient information and informed consent forms of this non-interventional study were approved by the ethics committees for the Department of Psychiatry and Psychotherapy, Semmelweis University in Budapest, Hungary and for the Prague Psychiatric Center/National Institute of Mental Health, Czech Republic. During the course of the study, a third study site (second Czech site) was added, Psychiatric Clinic University Hospital in Olomouc, in order to increase subjects' enrollment. The change was approved by the Ethics Committee.

Informed consent Informed consent was obtained from all individual participants included in the study.

Open Access This article is distributed under the terms of the Creative Commons Attribution 4.0 International License (http://creativeco mmons.org/licenses/by/4.0/), which permits unrestricted use, distribution, and reproduction in any medium, provided you give appropriate credit to the original author(s) and the source, provide a link to the Creative Commons license, and indicate if changes were made.

\section{References}

American Psychiatric Association (2000) Diagnostic and statistical manual of mental disorders (text revison, DSM-IV-TR), 4th edn. American Psychiatric Association, Washington
American Psychiatric Association (2013) Diagnostic and statistical manual of mental disorders, 5h edn. American Psychiatric Association, Arlington

Asherson P, Chen W, Craddock B, Taylor E (2007) Adult attentiondeficit hyperactivity disorder: recognition and treatment in general adult psychiatry. Br J Psychiatry 190:4-5

Asherson P, Young AH, Eich-Höchli D, Moran P, Porsdal V, Deberdt W (2014) Differential diagnosis, comorbidity, and treatment of attention-deficit/hyperactivity disorder in relation to bipolar disorder or borderline personality disorder in adults. Curr Med Res Opin 8:1657-1672

Balazs J, Kereszteny A (2017) Attention-deficit/hyperactivity disorder and suicide: a systematic review. World J Psychiatry 7:44-59

Balázs J, Bitter I, Hideg K, Vitrai J (1998) A MINI és a MINI Plusz kérdõív magyar nyelvû változatának kidolgozása. (Development of the Hungarian version of the MINI and MINI Plus questionnaires). Psychiatria Hung 13:160-168

Biederman J (2004) Impact of comorbidity in adults with attentiondeficit/hyperactivity disorder. J Clin Psychiatry 65(Suppl 3):3-7

Biederman J, Faraone SV, Spencer T, Wilens T, Norman D, Lapey KA et al (1993) Patterns of psychiatric comorbidity, cognition, psychosocial functioning in adults with attention deficit hyperactivity disorder. Am J Psychiatry 150:1792-1798

Biederman J, Ball SW, Monuteaux MC, Mick E, Spencer TJ, McCreary $M$ et al (2008) New insights into the comorbidity between ADHD and major depression in adolescent and young adult females. J Am Acad Child Adol Psychiatry 47:426-434

Bitter I, Simon V, Balint S, Meszaros A, Czobor P (2010) How do different diagnostic criteria, age and gender affect the prevalence of attention deficit hyperactivity disorder in adults? An epidemiological study in a Hungarian community sample. Eur Arch Psychiatry Clin Neurosci 260:287-296

Buitelaar JK, Kan CC, Asherson P (2011) ADHD in adults. Characterization, diagnosis, and treatment. Cambridge University Press, New York

Chang Z, Lichtenstein P, D’Onofrio BM, Sjölander A, Larsson H (2014) Serious transport accidents in adults with attention-deficit/ hyperactivity disorder and the effect of medication: a populationbased study. JAMA Psychiatry 71:319-325

Chen Q, Sjölander A, Runeson B, D'Onofrio BM, Lichtenstein P, Larsson H (2014) Drug treatment for attention-deficit/hyperactivity disorder and suicidal behaviour: register based study. BMJ 348:g3769. https://doi.org/10.1136/bmj.g3769

Conners CK, Erhardt D, Sparrow EP (1999) Conners' Adult ADHD Rating Scales (CAARS). Multi-Health Systems Inc, North Tonawanda

Cumyn L, French L, Hechtman L (2009) Comorbidity in adults with attention-deficit hyperactivity disorder. Can J Psychiatry 54:673-683

Deberdt W, Thome J, Lebrec J, Kraemer S, Fregenal I, Ramos-Quiroga JA, Arif M (2015) Prevalence of ADHD in nonpsychotic adult psychiatric care (ADPSYC): a multinational cross-sectional study in Europe. BMC Psychiatry 15:242

Doshi JA, Hodgkins P, Kahle J, Sikirica V, Cangelosi MJ, Setyawan J, Erder MH, Neumann PJ (2012) Economic impact of childhood and adult attention-deficit/hyperactivity disorder in the United States. J Am Acad Child Adolesc Psychiatry 51:990-1002

Fayyad J, Sampson NA, Hwang I, Adamowski T, Aguilar-Gaxiola S, Al-Hamzawi A et al (2017) The descriptive epidemiology of DSM-IV-TR adult ADHD in the World Health Organization world mental health surveys. Atten Defic Hyperact Disord 9:47-65

Ginsberg Y, Quintero J, Anand E, Casillas M, Upadhyaya HP (2014) Underdiagnosis of attention-deficit/hyperactivity disorder in adult patients: a review of the literature. Prim Care Companion CNS Disord 16:pii: PCC.13r01600 
Halmoy A, Halleland H, Dramsdahl M, Bergsholm P, Fasmer OB, Haavik J (2010) Bipolar symptoms in adult attention-deficit/ hyperactivity disorder: a cross-sectional study of 510 clinically diagnosed patients and 417 population-based controls. J Clin Psychiatry 71:48-57

Jacob CP, Romanos J, Dempfle A, Heine M, Windemuth-Kieselbach C, Kruse A et al (2007) Co-morbidity of adult attention-deficit/ hyperactivity disorder with focus on personality traits and related disorders in a tertiary referral center. Eur Arch Psych Clin Neurosci 257:309-317

Kessler RC, Adler L, Ames M, Demler O, Faraone S, Hiripi EVA et al (2005) The world health organization adult ADHD self-report scale (ASRS): a short screening scale for use in the general population. Psychol Med 35:245-256

Kessler RC, Adler LA, Gruber MJ, Sarawate CA, Spencer T, Van Brunt DL (2007) Validity of the World Health organization adult ADHD self-report scale (ASRS) screener in a representative sample of health plan members. Int J Methods Psychiatr Res 16(2):52-65

Kooij SJ, Bejerot S, Blackwell A, Caci H, Casas-Brugué M et al (2010) European consensus statement on diagnosis and treatment of adult ADHD: the European network adult ADHD. BMC Psychiatry 10:67

Lichtenstein P, Halldner L, Zetterqvist J, Sjölander A, Serlachius E, Fazel S, Långström N, Larsson H (2012) Medication for attention deficit-hyperactivity disorder and criminality. N Engl J Med 367:2006-2014

Matte B, Rohde LA, Turner JB, Fisher PW, Shen S, Bau CH (2015) Reliability and validity of proposed DSM-5 ADHD symptoms in a clinical sample of adults. J Neuropsychiatry Clin Neurosci 27:228-236

Ohlmeier MD, Peters K, Wildt BTT, Zedler M, Ziegenbein M, Wiese B, Emrich HM, Schneider U (2008) Comorbidity of alcohol and substance dependence with attention-deficit/hyperactivity disorder (ADHD). Alcohol Alcohol 43:300-304

Perroud N, Cordera P, Zimmermann J, Michalopoulos G, Bancila V, Prada P, Dayer A, Aubry JM (2014) Comorbidity between attention deficit hyperactivity disorder (ADHD) and bipolar disorder in a specialized mood disorders outpatient clinic. J Affect Disord 168:161-166

Ramos-Quiroga JA, Montoya A, Kutzelnigg A, Deberdt W, Sobanski E (2013) Attention deficit hyperactivity disorder in the European adult population: prevalence, disease awareness and treatment guidelines. Curr Med Res Opin 29:1093-1104
Rao P, Place M (2011) Prevalence of ADHD in four general adult outpatient clinics in North East England. Prog Neurol Psychiatry 15:7-10

Sheehan DV, Lecrubier Y, Sheehan KH, Amorim P, Janavs J, Weiller $E$ et al (1998) The mini-international neuropsychiatric interview (M.I.N.I.): the development and validation of a structured diagnostic psychiatric interview for DSM-IV and ICD-10. J Clin Psychiatry 59:22-33

Sibley MH, Rohde LA, Swanson JM, Hechtman LT, Molina BS, Mitchell JT et al (2018) Late-onset ADHD reconsidered with comprehensive repeated assessments between ages 10 and 25. Am J Psychiatry 175(2):140-149

Simon V, Czobor P, Balint S, Meszaros A, Bitter I (2009) Prevalence and correlates of adult attention-deficit hyperactivity disorder: meta-analysis. Br J Psychiatry 194:204-211

Simon V, Czobor P, Bitter I (2013) Is ADHD severity in adults associated with the lifetime prevalence of comorbid depressive episodes and anxiety disorders? Eur Psychiatry 28:308-314

Sobanski E, Bruggemann D, Alm B, Kern S, Deschner M, Schubert T et al (2007) Psychiatric comorbidity and functional impairment in a clinically referred sample of adults with attention-deficit/hyperactivity disorder (ADHD). Eur Arch Psychiatry Clin Neurosci 257:371-377

Sonuga-Barke EJ (2005) Causal models of attention-deficit/hyperactivity disorder: from common simple deficits to multiple developmental pathways. Biol Psychiatry 57:1231-1238

Spencer TJ, Biederman J, Mick E (2007) Attention-deficit/hyperactivity disorder: diagnosis, lifespan, comorbidities, and neurobiology. J Pediatr Psychol 32:631-642

Sprafkin J, Gadow KD, Weiss MD, Schneider J, Nolan EE (2007) Psychiatric comorbidity in ADHD symptom subtypes in clinic and community adults. J Atten Dis 11:114-124

Vitola ES, Bau CHD, Salum GA, Horta BL, Quevedo L, Barros FC et al (2017) Exploring DSM-5 ADHD criteria beyond young adulthood: phenomenology, psychometric properties and prevalence in a large three-decade birth cohort. Psychol Med 47:744-754

Wilens TE, Biederman J, Faraone SV, Martelon M, Westerberg D, Spencer TJ (2009) Presenting ADHD symptoms, subtypes, and comorbid disorders in clinically referred adults with ADHD. J Clin Psychiatry 70:1557-1562 